\title{
Three-Dimensional Analysis of Deep Space Network Antenna Coverage
}

\author{
Obadiah Kegege \\ NASA Glenn Research Center \\ 21000 Brookpark Rd. \\ Cleveland, OH 44135 \\ 216-433-3127 \\ obadiah.o.kegege@nasa.gov
}

\author{
Michael Fuentes \\ QinetiQ North America. \\ NASA Glenn Research Center \\ 21000 Brookpark Rd. \\ Cleveland, OH 44135 \\ michael.a.fuentes@nasa.gov
}

\author{
Nicholas Meyer, Amy Sil \\ NASA Glenn Research Center \\ 21000 Brookpark Rd. \\ Cleveland, OH 44135 \\ nicholas.e.meyer@nasa.gov, \\ amy.a.sil@nasa.gov
}

\begin{abstract}
There is a need to understand NASA's Deep Space Network (DSN) coverage gaps and any limitations to provide redundant communication coverage for future deep space missions, especially for manned missions to Moon and Mars. The DSN antennas are required to provide continuous communication coverage for deep space flights, interplanetary missions, and deep space scientific observations. The DSN consists of ground antennas located at three sites: Goldstone in USA, Canberra in Australia, and Madrid in Spain. These locations are not separated by the exactly 120 degrees and some DSN antennas are located in the bowl-shaped mountainous terrain to shield against radiofrequency interference resulting in a coverage gap in the southern hemisphere for the current DSN architecture. To analyze the extent of this gap and other coverage limitations, simulations of the DSN architecture were performed. In addition to the physical properties of the DSN assets, the simulation incorporated communication forward link calculations and azimuth/elevation masks that constrain the effects of terrain for each DSN antenna. Analysis of the simulation data was performed to create coverage profiles with the receiver settings at a deep space altitudes ranging from 2 million to 10 million $\mathbf{k m}$ and a spherical grid resolution of 0.25 degrees with respect to longitude and latitude. With the results of these simulations, two- and three-dimensional representations of the area without communication coverage and area with coverage were developed, showing the size and shape of the communication coverage gap projected in space. Also, the significance of this communication coverage gap is analyzed from the simulation data.
\end{abstract}

\section{TABLE OF CONTENTS}

1. INTRODUCTION ................................................1

2. OVERVIEW OF THE PRESENT DSN...................1 3. STUDY APPROACH AND MODEL

PARAMETERS .....................................................2

\section{ANALYSIS OF DSCC TERRAIN AND AZ-EL}

MASKS

5. DSN COMMUNICATION COVERAGE SIMULATION DATA.................................................4

6. SUMMARY ........................................................8

REFERENCES...............................................8

BIOGRAPHIES...................................................8

U.S. Government work not protected by U.S. copyright

\section{INTRODUCTION}

This paper presents the analysis of NASA's Deep Space Network (DSN) communication coverage. In the history of space exploration, DSN antennas have provided communication coverage of spacecrafts and satellites from Geosynchronous Earth Orbit (GEO) to the edge of our solar system. Because of the location of DSN antennas, a communication coverage gap exists in the southern hemisphere. There is a need to understand this communication gap to assess the possible impact on current and future space missions.

This communication coverage gap was analyzed using NASA's Integrated SCaN (Space Communication and Navigation) Simulator. The SCaN model library was used to develop the physical attributes and constraints of the DSN architecture within the simulation. In the analysis, we created models of DSN antennas (as presented in section 3) and receiver models at various deep space altitudes ranging up to 10 million $\mathrm{km}$. The simulations incorporated antenna parameters, forward link calculations, and the azimuth/elevation terrain masks to constrain the line of sight for each antenna. Also, the SCaN model library and Satellite Tool Kit (STK) were used to develop the simulations.

From the results of the simulations, two-dimensional (2D) and three-dimensional (3D) profiles representing the DSN communication coverage are presented in this paper. Also, the profiles show the size, shape, and the percentage of the communication coverage gap projected in space at various deep space altitudes.

\section{Overview of The Present DSN}

The DSN comprises three Deep Space Communications Complex (DSCC) facilities that are located in Goldstone, USA; Madrid, Spain; and Canberra, Australia. Each DSCC has one 70-m antenna, one 34-m high efficiency (HEF) antenna, at least one 34-m beam wave guide (BWG) antenna, and a signal processing center [1]. Also, the DSN has test facilities that support compatibility testing (Figure 1): Development Test Facility (DTF-21) that conducts tests of radiofrequency (RF) compatibility between the DSN and the customer's flight system, Merritt Island Launch (MIL71) support facility that provides DSN-compatible support 


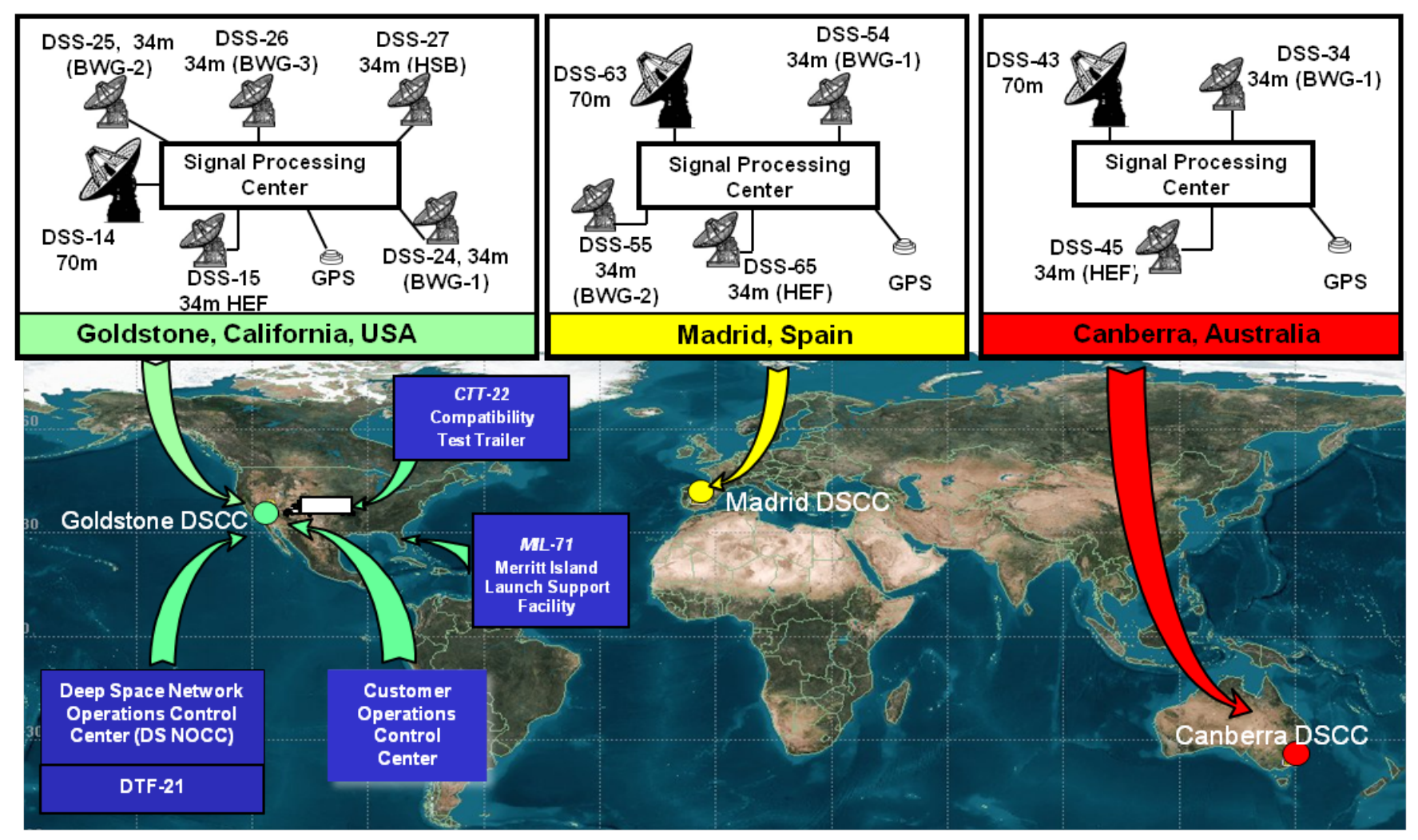

Figure 1. Overview of DSN

for launch operations, and a transportable Compatibility Test Trailer (CTT-22) that conducts RF compatibility tests between the DSN and the customer's flight system at the customer's facility [1].

\section{Study Approach AND MOdel PARAMEters}

Figure 1 shows the 13 antennas that are currently operated by DSN. Each of these antennas was modeled as a transmitter. To perform forward link calculations, receivers were modeled at various deep space altitudes (simulated up to 10 million $\mathrm{km}$ ). For each DSN antenna, we used the specific antenna parameters and terrain data to define the simulation scenarios. These parameters include the position using the Cartesian coordinates of DSN antennas in the ITRF93 reference system [2], antenna size and shape, frequency bands, polarization, transmitting power, operating azimuth/elevation (Az/El) angles, half-power beamwidth, and main-lobe gain [3], [4], [5], and [6].

Other parameters such as the effective isotropic radiated power (EIRP) are calculated in the simulations. The effective isotropic radiated power is given as:

$$
\mathrm{EIRP}=P_{t}+G_{t}
$$

where $P_{t}$ is the power of the transmitting antenna and $G_{t}$ is the gain of the transmitting antenna. At deep space receiver grid points, the received power is approximated by $P_{r}=\operatorname{EIRP}-L_{p}+G_{r}$, and can be rewritten as:

$$
P_{r}=\operatorname{EIRP}+G_{r}+20 \log \left(\frac{c}{4 \pi f}\right)-20 \log (R)
$$

where

$L_{p}=20 \log (R)-20 \log \left(\frac{c}{4 \pi f}\right)$ is the propagation path loss, $R$ is the distance between transmitter and receiver, $G_{r}$ is the gain of the receiver, $C$ is the speed of light, and $f$ is the frequency.

\begin{tabular}{|c|c|c|}
\hline & $\begin{array}{c}\text { Canberra } \\
\text { DSS } 34 \\
\text { X-Band Transmitter }\end{array}$ & $\begin{array}{c}\text { Goldstone } \\
\text { DSS } 14 \\
\text { X-Band Transmitter }\end{array}$ \\
\hline $\begin{array}{l}\text { Position } \\
\text { (m) }\end{array}$ & $\begin{array}{c}X=-4461146.756 \\
Y=+2682439.293 \\
Z=-3674393.542\end{array}$ & $\begin{array}{l}X=-2353621.251 \\
Y=-4641341.542 \\
Z=+3677052.370\end{array}$ \\
\hline $\begin{array}{l}\text { Frequency } \\
(\mathrm{GHz})\end{array}$ & 7.145 & 7.145 \\
\hline Antenna type & Parabolic Reflector & Parabolic Reflector \\
\hline Polarization & RCP/LCP & RCP/LCP \\
\hline Diameter (m) & 34 & 70 \\
\hline $\begin{array}{c}\text { Elevation } \\
\text { angle (deg) }\end{array}$ & $\begin{array}{c}\text { Min: } 5.923 \\
\text { Max: } 89.577\end{array}$ & $\begin{array}{c}\text { Min: } 5.962 \\
\text { Max: } 89.538\end{array}$ \\
\hline Power (W) & 20,000 & 20,000 \\
\hline Gain (dBi) & 68.3 & 74.5 \\
\hline
\end{tabular}

Table 1. Example of parameters used in the models 
The transmit parameters (as expressed in Table 1) for each antenna were incorporated into the simulation scenario. The minimum and maximum elevation angles in Table 1 define the mechanical limits of the antenna. The line-of-sight of each antenna, which affects the overall coverage, is also affected by the surrounding terrain. Given the line-of-sight characteristics, a given area in space may have redundant coverage from two or three DSCC antennas.

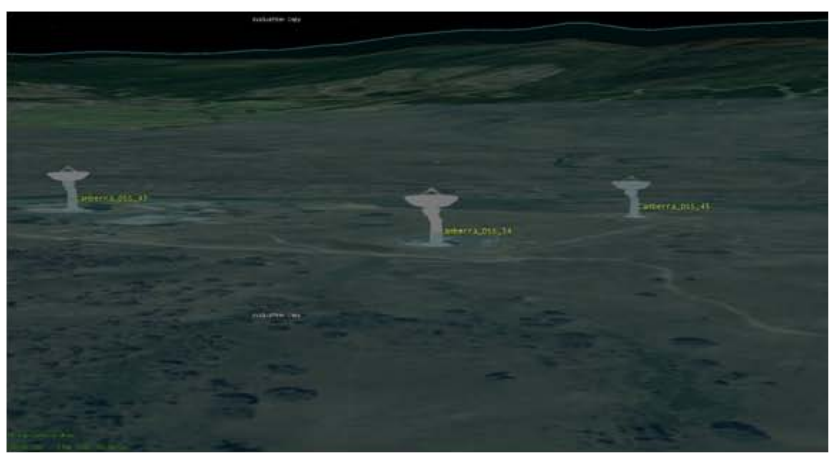

Canberra DSCC

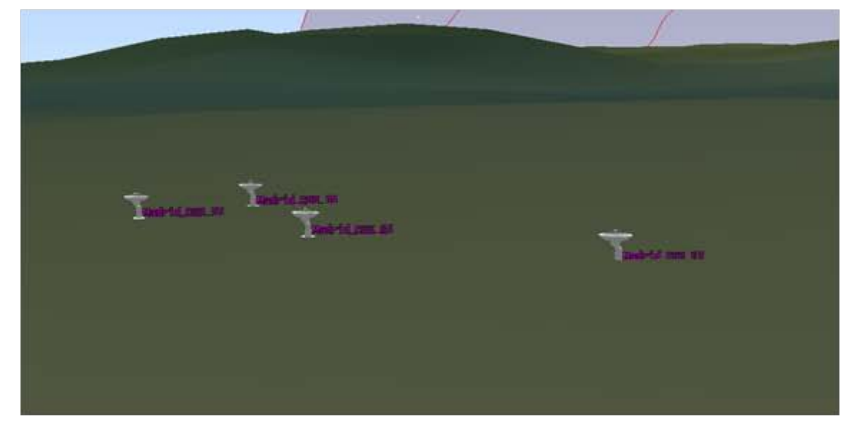

Madrid DSCC

\section{ANALYSIS OF DSCC TERRAIN AND AZ-EL MASKS}

Figure 2 shows the terrain for Canberra Deep Space Communications Complex (CDSCC), Goldstone Deep Space Communications Complex (GDSCC), Madrid Deep Space Communications Complex (MDSCC), and the Az-El mask for each DSCC.

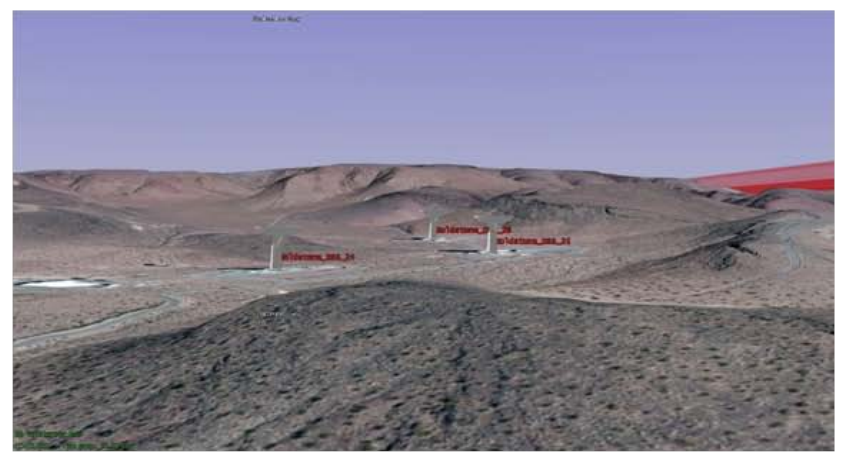

Goldstone DSCC

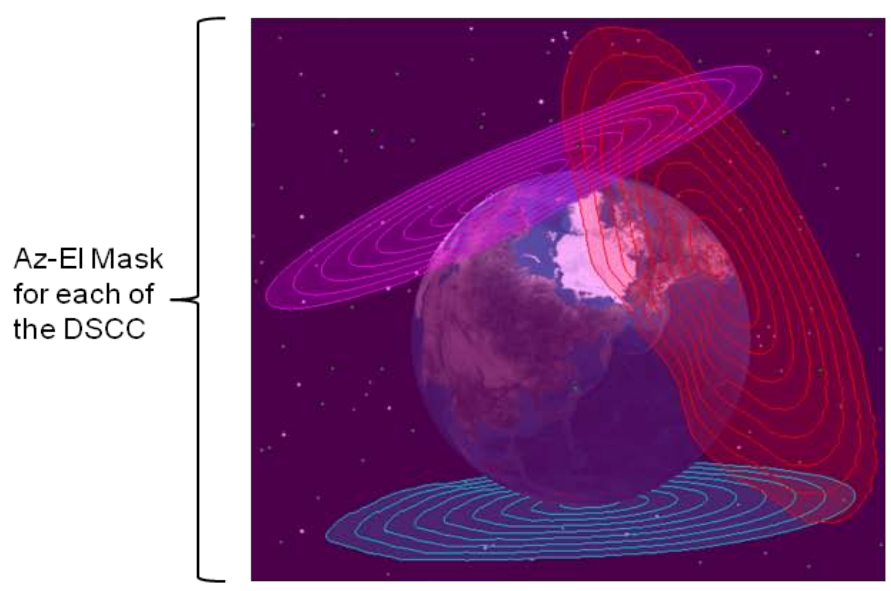

Figure 2. DSN terrain and Az-El masks for each DSCC

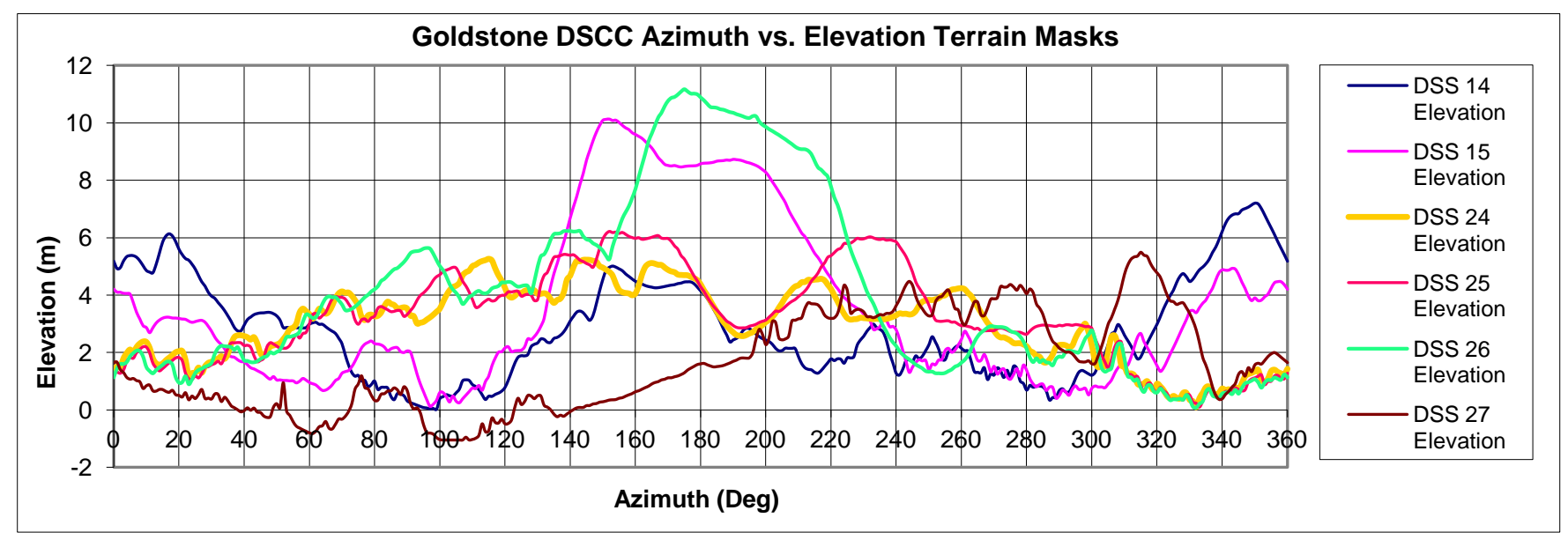

Figure 3. Terrain for each Deep Space Station (DSS) antenna at Goldstone DSCC 
The terrain for GDSCC has a resolution of $1 / 9$ arc-second $(\sim 3 \mathrm{~m})$, where as CDSCC and MDSCC have 1 arc-second $(\sim 30 \mathrm{~m})$ resolution of terrain data. Using this terrain data, the Az-El masks shown in Figure 2 were computed (i.e., in Figure 3 for GDSCC). Each Deep Space Station (DSS) antenna's line of sight is evaluated against the terrain during visibility computations (Figure 3). These masks serve to model the physical obstruction of the area surrounding the antennas, which is necessary for determining the communication coverage gap.

\section{DSN COMMUNICATION COVERAGE SiMULATION DATA}

Several simulations were performed using Satellite Took Kit (STK) and other tools. Data from these computations were used to create 2D and 3D communication coverage profiles with the receiver settings at deep space altitudes ranging up to 10 million $\mathrm{km}$ with a spherical grid resolution of 0.25 degrees with respect to longitude and latitude. The coverage area of each individual antenna is generated and merged to produce the global coverage provided by the DSN at that various altitudes. Figures 4 (a) and (b) show 3D communication coverage profile, representing the size and shape of the gap projected in space. The area shown in pink (Figure 4) has communication coverage from at least one DSN antenna. The area shown in black is the communication coverage gap.

Figure 5, 6, 7, and 8 show 2D coverage profiles projected up to altitudes of 40,000 km, 400,000 km, 4 million km, and 10 million $\mathrm{km}$. These profiles show the projection of the combined coverage on the Earth's surface. A comparison of the $2 \mathrm{D}$ projections of the coverage gap for altitudes of $40,000 \mathrm{~km}$ (Figure 5) and 400,000 km (Figure 6) show that the communication coverage gap decreases with increase in altitude. However, a comparison of the altitudes at 4,000,000 km (Figure 7) and 10,000,000 km (Figure 8) show little change. The exponential decay of this gap with altitude is analyzed in section 5 .

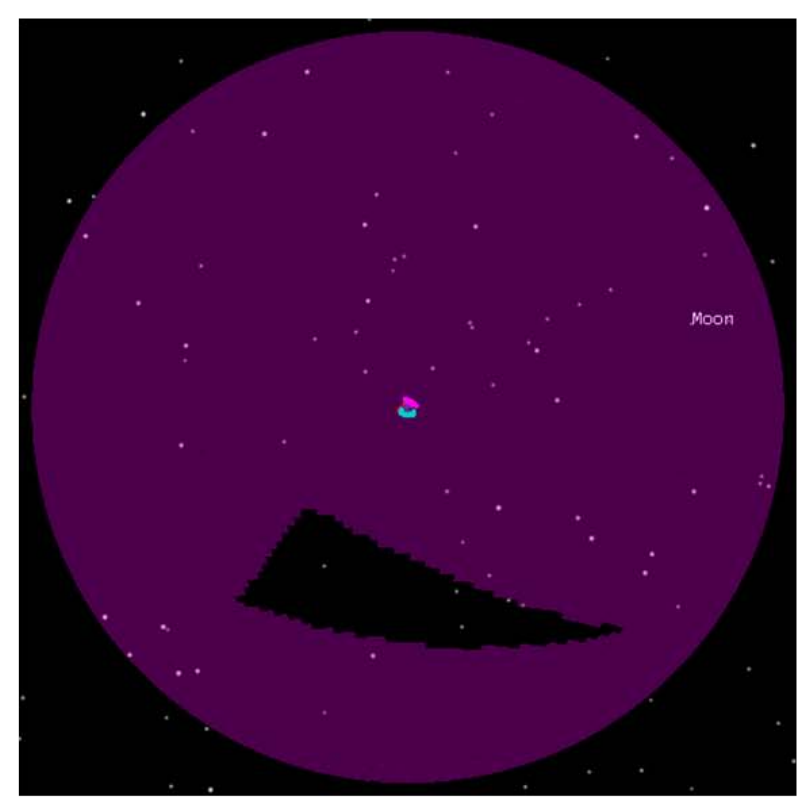

(b) DSN coverage at altitude of $400,000 \mathrm{~km}$

(a) DSN coverage at altitude of $40,000 \mathrm{~km}$

Figure 4. 3D profiles of DSN communication coverage 


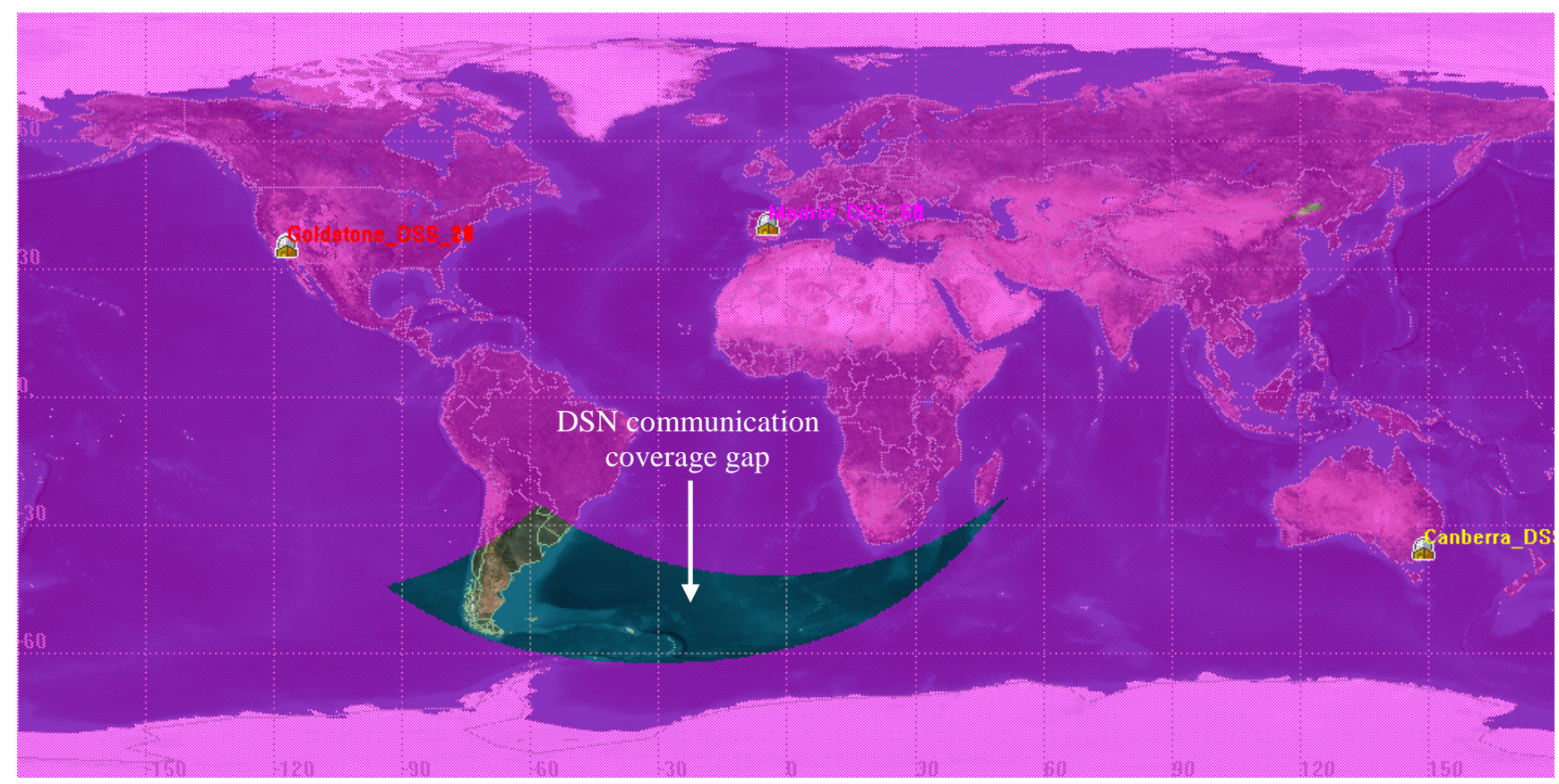

Figure 5. 2D profile of DSN communication coverage at altitude of $40,000 \mathrm{~km}$

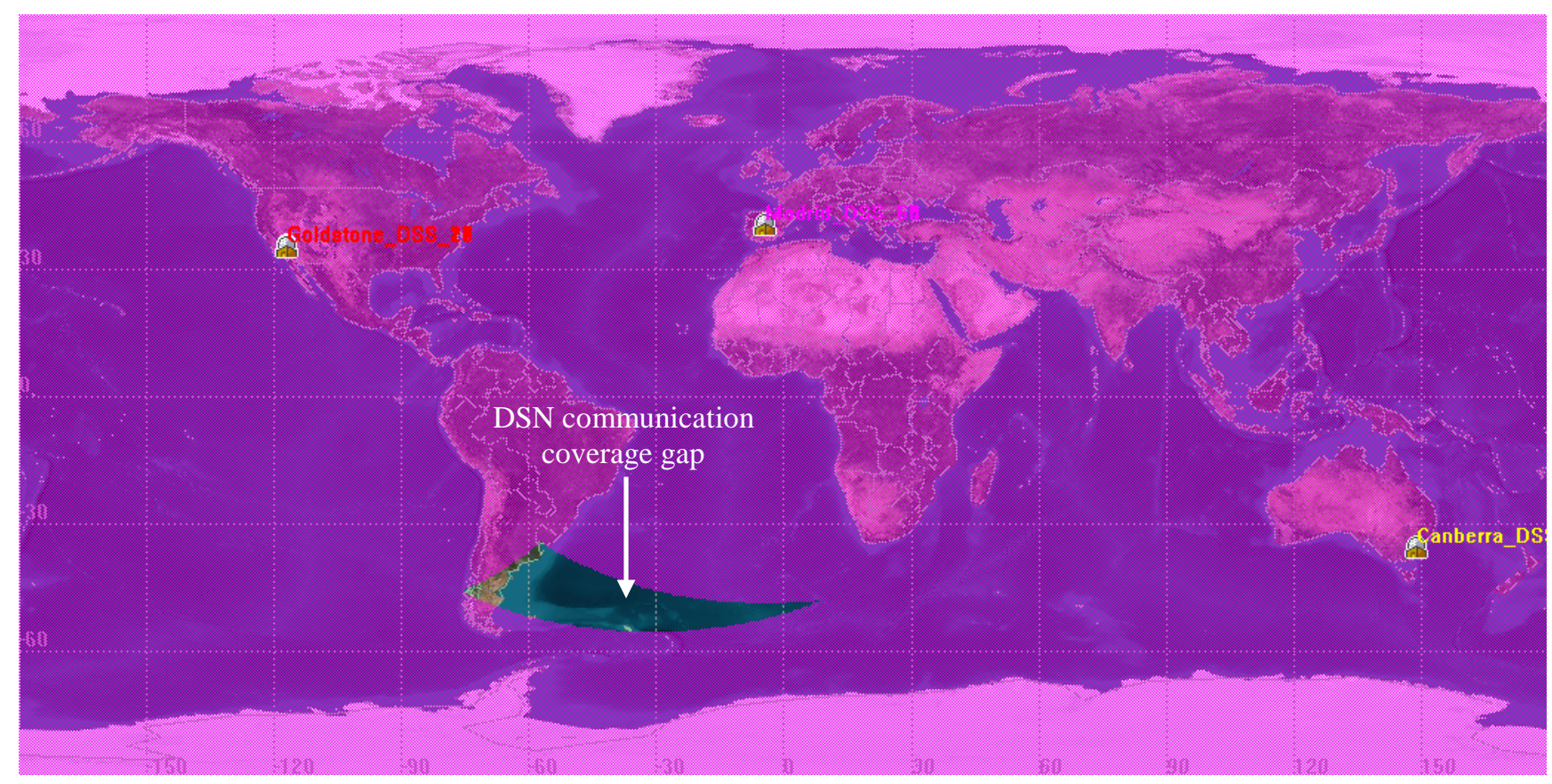

Figure 6. 2D profile of DSN communication coverage at altitude of $400,000 \mathrm{~km}$ 


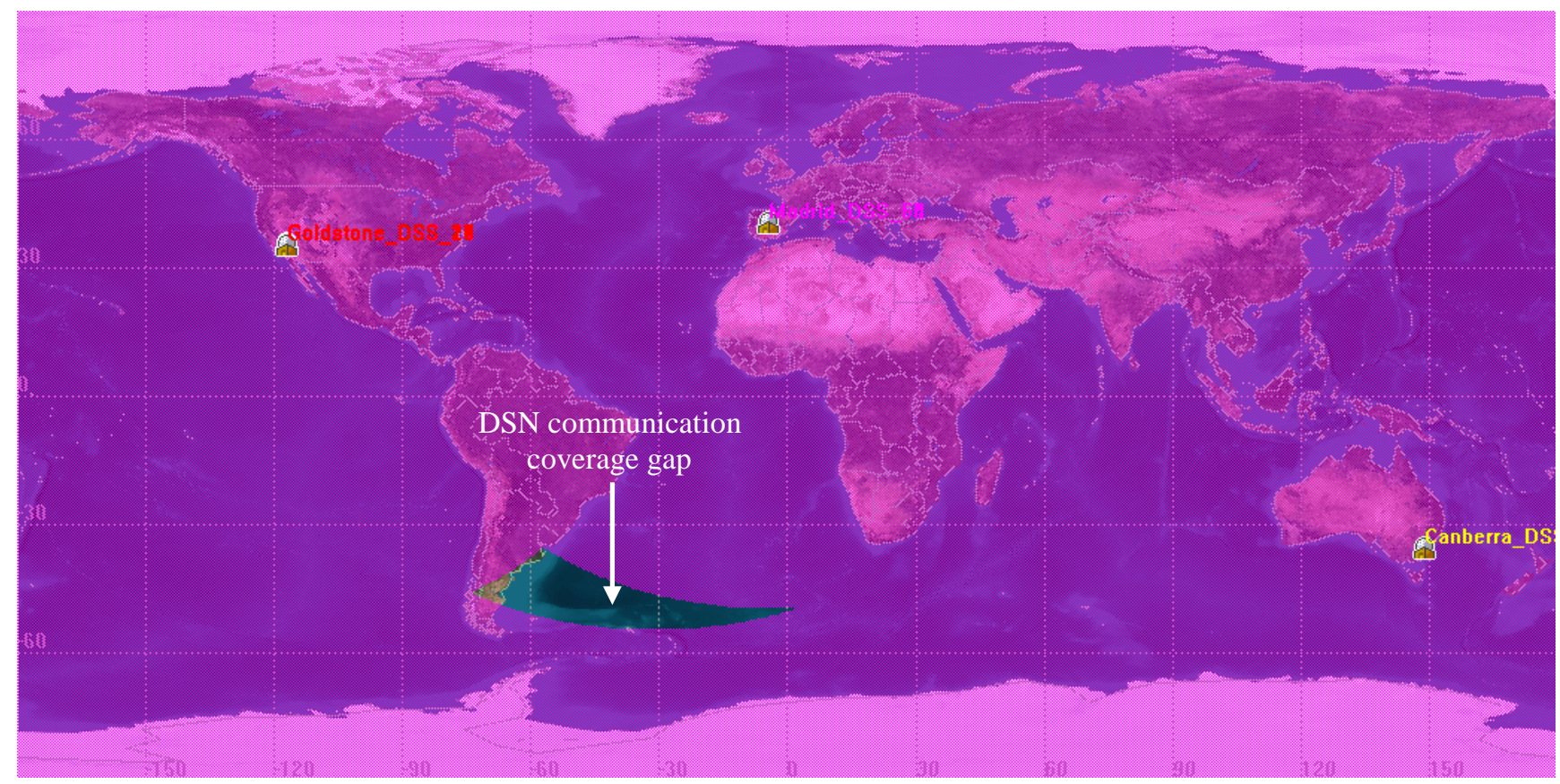

Figure 7. 2D profile of DSN communication coverage at altitude of $4,000,000 \mathrm{~km}$

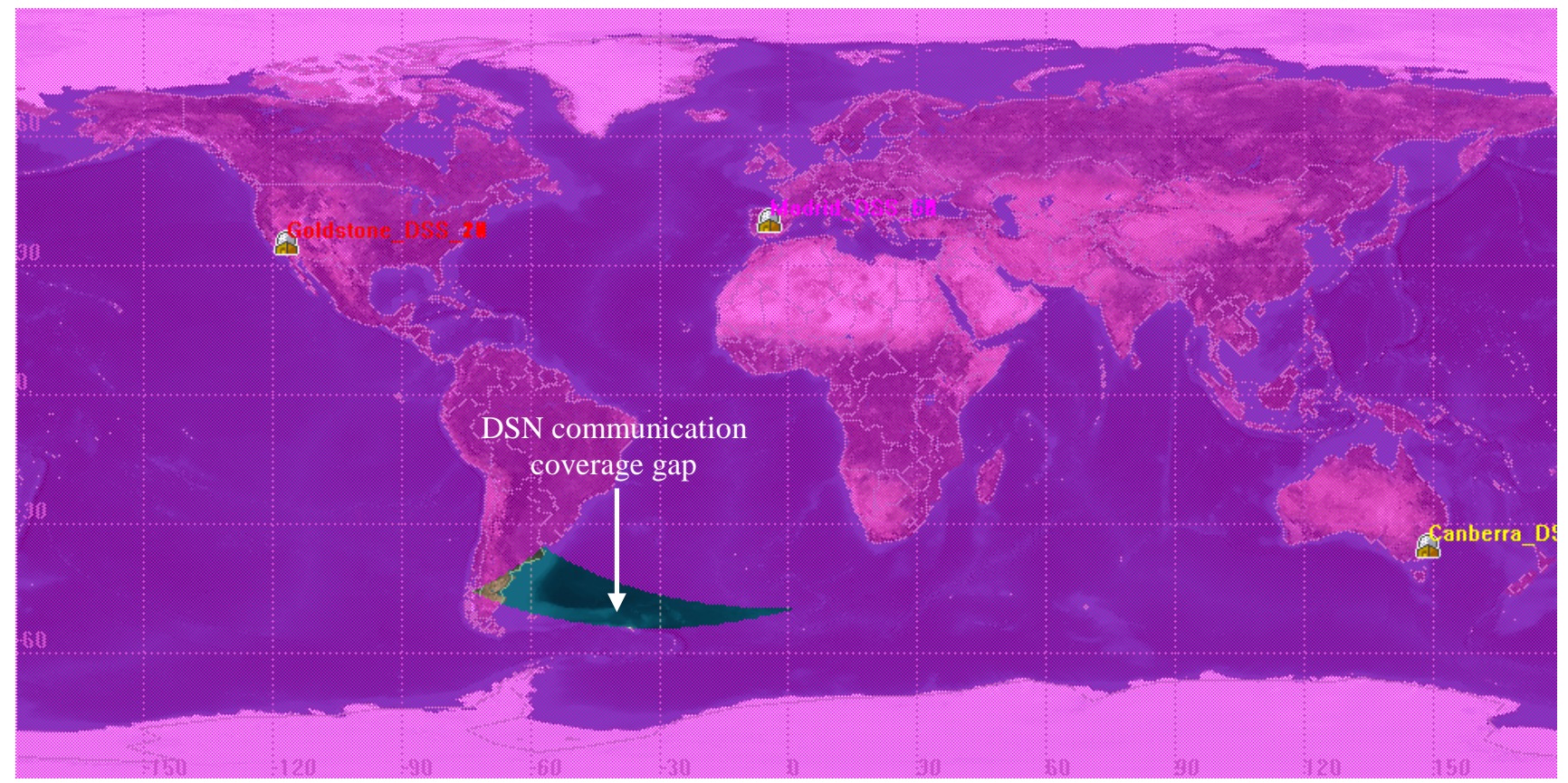

Figure 8. 2D profile of DSN communication coverage at altitude of 10,000,000 km

Figure 9 graphically shows the relationship between the percentage of the area with DSN communication and altitude of the corresponding receiver. The altitudes range from 2 million (distance from the Earth's surface where deep space begins [7]) to 10 million $\mathrm{km}$ (maximum value allowed by the simulation software). By looking at the DSN coverage gap in Figures 4, 5, 6, 7, and 8; then comparing with the plot in Figure 9, we can see that coverage area increases rapidly then increases at a slower pace. This is a logarithmic function that can be predicted to have a very minimal increase after 10 million $\mathrm{km}$. From analyzing the data in Figure 9, a function representing the percentage of the current DSN coverage at altitudes of greater than 2 million $\mathrm{km}$ can be approximated as:

$$
\mathrm{DSN}_{\% \text { coverage }}=99.078+m \ln (R-1)
$$


where $R$ (greater or equal to 2 ) is the altitude (in millions of $\mathrm{km})$ and $m$ is a function of the altitude and other parameters discussed in sections 3 and 4 .

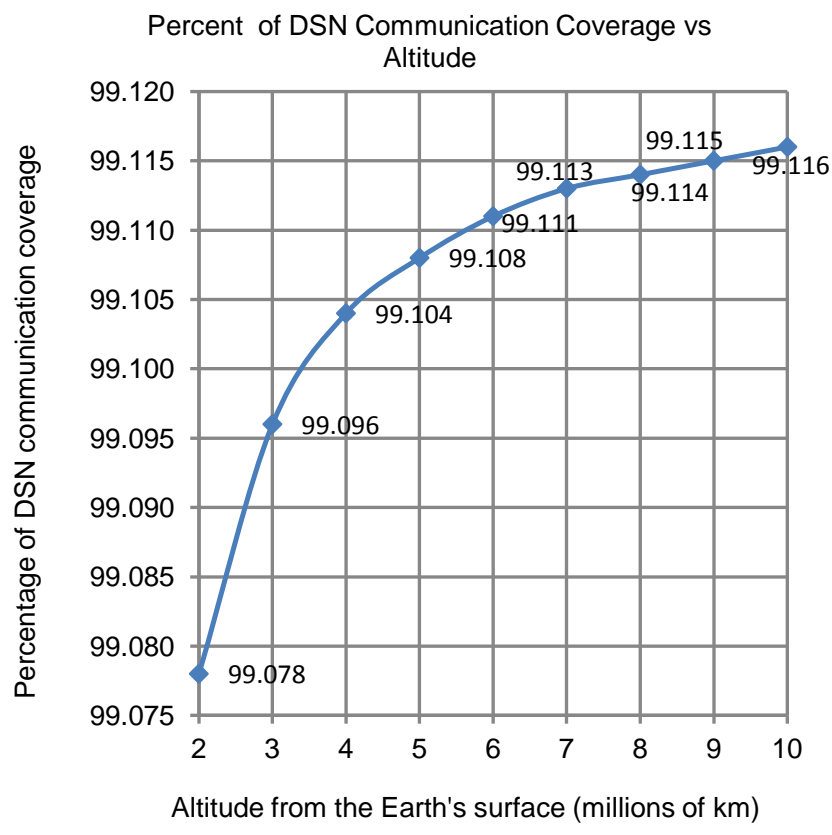

\section{Figure 9. Percentage of DSN communication coverage by altitude}

Using Equation 3 to solve a system of equations for the data points in Figure 9, an equation representing the percentage of the current DSN communication coverage for altitudes of greater than 2 million $\mathrm{km}$ was derived as:

$$
\mathrm{DSN}_{\% \text { coverage }}=99.078+\left(\frac{0.391+0.1215 R}{9.21 R}\right) \ln (R-1)
$$

whereby $m$ that was introduced in equation 3 is:

$$
m=\left(\frac{0.391+0.1215 R}{9.21 R}\right)
$$

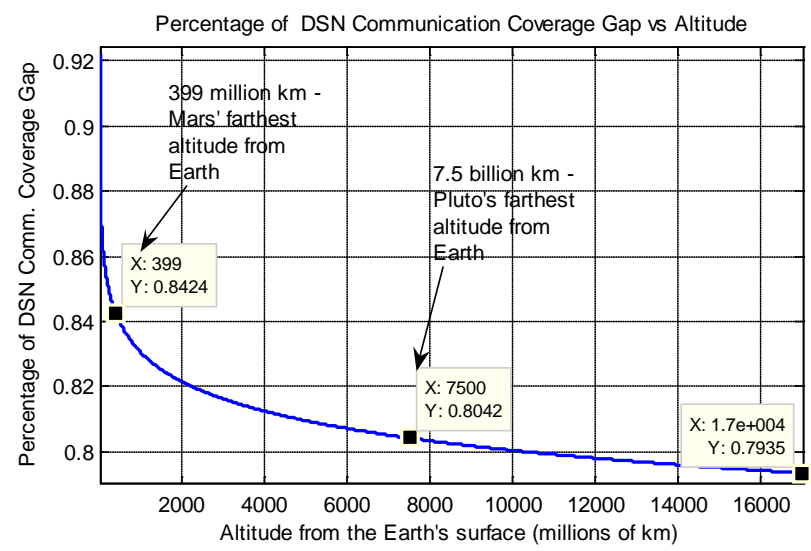

Figure 10. Percentage of DSN communication coverage gap by altitude
At an altitude of 17 billion km, more than twice Pluto's farthest (7.5 billion $\mathrm{km}$ ) orbiting altitude from Earth, the calculations show that DSN will have a coverage gap of $\sim 0.793 \%$ (Figure 10). The formulation in Equation 4 is limited to the current DSN antenna parameters and terrain data.

The full impact of the coverage gap cannot be assessed with static 2D analyses. The rotation of the Earth impacts where in space the gap is pointing. The location of the DSN gap can be formulated as in Figure 11. From Figure 11, we can see that the circumference of the Earth at the equator $C_{R}$ can be approximated as:

$$
C_{R}=2 \times \pi \times 6,378,137 \mathrm{~m}=40,075,016.69 \mathrm{~m}
$$

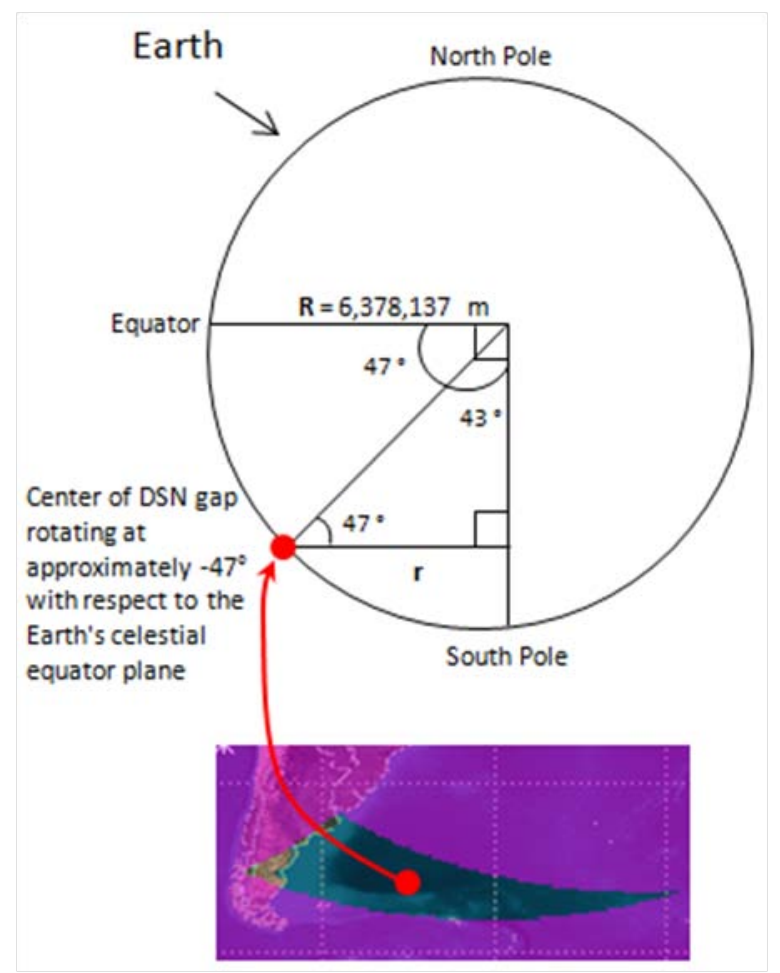

Figure 11. DSN gap and relative speed of Earth's rotation

The period of rotation of the Earth is 23 hours, 56 minutes, 4 seconds (86,164 s). The Earth's rotational velocity at the equator is:

$$
V_{R}=\frac{40,075,016.69 \mathrm{~m}}{86,164 \mathrm{~s}}=465.1 \mathrm{~m} / \mathrm{s}
$$

Since $r=\left(C_{R} / 2 \pi\right)\left(\cos \left(47^{\circ}\right)\right)=R \cos \left(47^{\circ}\right)$, at a point on the DSN coverage gap, this rotational speed can be approximated by multiplying the speed at the equator by the cosine of the angle where the DSN communication gap falls.

$$
V_{r}=465.1 \mathrm{~m} / \mathrm{s} \times \cos \left(47^{\circ}\right)=317.2 \mathrm{~m} / \mathrm{s}
$$


At an altitude of $10,000,000 \mathrm{~km}$, this gap stretches from the longitude of about $-75^{\circ}$ to $2^{\circ}$ (Figure 8). As the Earth rotates with the DSN communication gap rotating at 317.2 $\mathrm{m} / \mathrm{s}$, a space mission within this DSN communication coverage gap will be without coverage for a maximum of:

$$
\frac{77^{\circ}}{360^{\circ}} \times 86164 \mathrm{~s}=18430 \mathrm{~s}=5.12 \mathrm{hr}
$$

We have seen that, at an altitude of $10,000,000 \mathrm{~km}$, a mission can be without coverage for up to 5.12 hours if it falls in the DSN coverage gap. This phenomenon is demonstrated in Figure 12.

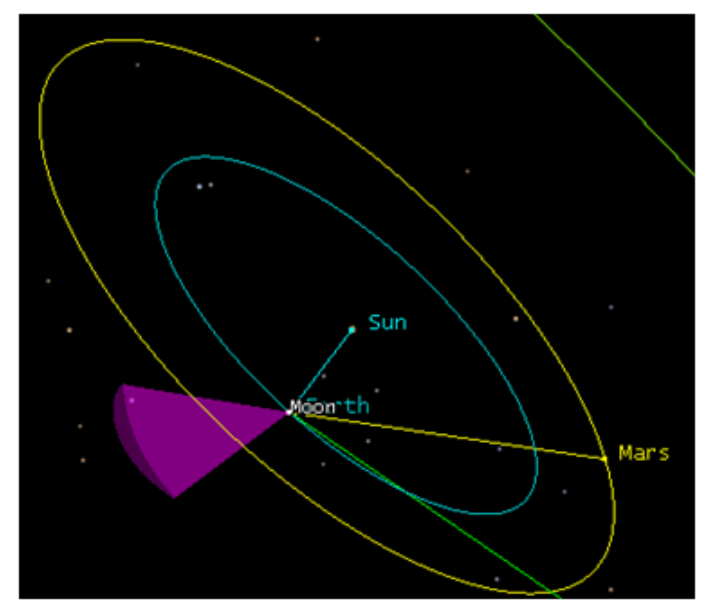

Figure 12. 3D simulation of DSN communication coverage gap projected in space

The planetary bodies in the Solar System lie near the ecliptic plane. The ecliptic plane is tilted $23.5^{\circ}$ with respect to the Earth's celestial equator plane since the Earth's rotational axis is tilted $23.5^{\circ}$ because of this none of the eight solar system planets fall within this DSN coverage gap. Pluto (dwarf planet) is inclined $\sim 17^{\circ}$ relative to the ecliptic plane but does not get in the DSN coverage gap.

\section{SUMMARY}

In this study, we have modeled the DSN antenna parameters and communication link, and incorporated the terrain constraints to evaluate the communication coverage gaps within the DSN. In addition to each of the DSN antenna's mechanical constraints and radiation pattern, terrain data was incorporated to model the communication coverage gap within DSN. From the simulation data, we have presented both 2D and 3D communication coverage profiles showing the location, size, and shape of this communication coverage gap projected in space at various deep space altitudes.

The deep space antennas are required to provide coverage of deep space missions throughout the solar system. Though our simulations were limited to an altitude of 10,000,000 $\mathrm{km}$, a formula was derived that modeled the percentage of the current DSN coverage for deep space altitudes of greater than 10,000,000 km. This analysis was based on the parameters of the current DSN assets. The analysis can be used to assess the amount of time a mission can be without communication coverage at any altitude if it falls within this DSN gap. However, none of the current missions fall within the DSN communication coverage gap. This study did not include all celestial bodies in the solar system or outside the solar system. Therefore, authors recommend analyzing the trajectories of Potentially Hazardous Asteroids (PHAs) to assess the capabilities of DSN antennas supporting a PHA mission.

\section{REFERENCES}

[1] JPL/NASA, "Deep Space Network Services Catalog," DSN No. 820-100, Rev. E, Issued December 17, 2009.

[2] JPL, "Cartesian coordinates of DSN antennas in the ITRF93 reference system (International Terrestrial Reference Frame),” http://dsnra.jpl.nasa.gov/Antennas/ Antennas.html

[3] JPL/NASA, “34-m and 70-m Command,” DSN No. 205, Rev. C, Released June 1, 2010.

[4] JPL/NASA, "34-m BWG Antennas Telecommunications Interfaces,” DSN No. 104, Rev. F, Released June 1, 2010.

[5] JPL/NASA, “34-m HEF Subnet Telecommunications Interfaces,” DSN No. 103, Rev. B, September 19, 2008.

[6] JPL/NASA, "70-m Subnet Telecommunications Interfaces,” DSN No. 101, Rev. D, April 21, 2011.

[7] JPL/NASA, "Frequency and Channel Assignments," DSN No. 201, Rev. B, December 15, 2009.

\section{BIOGRAPHIES}

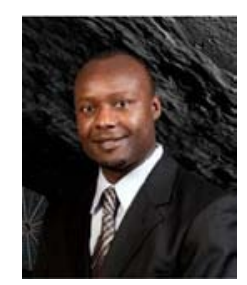

Obadiah Kegege is an Electronics Engineer in the Systems Definition and Communications Branch at NASA Glenn Research Center. He is part of the Space Communications and Navigation (SCaN) team. He received his BS in Control and Instrumentation Electronics Design from the University of Houston, MS in Electrical Engineering from the University of Texas Pan American, and a PhD from the University of Arkansas.

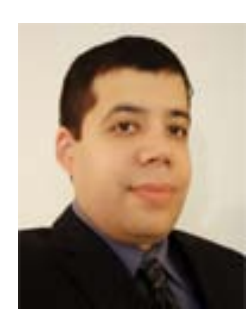

Michael Fuentes is a Space Communication Network Architect and private consultant at the NASA Glenn Research Center, where he is responsible for the modeling and simulation of space communication networks and systems. Prior to NASA, Michael worked at Custom Computer Services developing custom business software for produce and environmental regulations companies. Michael holds a Master's degree in computer engineering from Case 
Western Reserve University and a Bachelor's degree in computer engineering from California State University, Fresno.

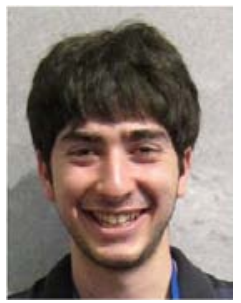

Nicholas Meyer is pursuing a B.S.E. in Computer Science at the University of Pennsylvania. He worked as an intern with the SCaN Summer Intern Program at the Glenn Research Center during the summer of 2011.

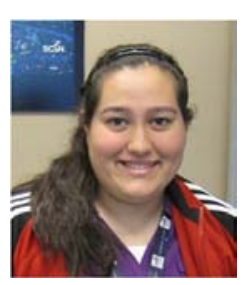

Amy Sil studying B.M.E at the University of Findlay. She worked as an intern with the SCaN Summer Intern Program at the Glenn Research Center during the summer of 2011. 